Physica 13D (1984) 195-210

North-Holland, Amsterdam

\title{
SINGULAR PERTURBATIONS AND A MAPPING ON AN INTERVAL FOR THE FORCED VAN DER POL RELAXATION OSCILLATOR
}

\author{
J. GRASMAN, H. NIJMEIJER* and E.J.M. VELING** \\ Centre for Mathematics and Computer Science, Kruislaan 413, 1098 SJ Amsterdam, The Netherlands
}

Received 15 April 1982

Revised 19 December 1983

\begin{abstract}
This paper deals with the Van der Pol relaxation oscillator with a large sinusoidal forcing term. By using singular perturbation techniques asymptotic solutions of such a system are constructed. These asymptotic approximations are locally valid and may take the form of a two time scale expansion in one region and a boundary layer type of solution in a next region. Integration constants are determined by averaging and matching conditions. From these local solutions a difference equation is constructed. There is an equivalence between solutions of the difference equation being an iterated mapping on a compact interval and solutions of the system itself. This equivalence makes it possible to analyze subharmonics and chaotic type of solutions to the full extent. As a result of this we find domains in the parameter space, where regular subharmonics exist. These domains overlap so that for some parameter values different subharmonics coexist. For parameter values in this range chaotic type of solutions are found as well. They are described by using concepts of symbolic dynamics.
\end{abstract}

\section{Introduction}

Recent studies of difference equations [14, 18, 25 ] have revealed unexpected phenomena which in literature are characterized by the term "chaotic". Some of these equations are meant to model biological phenomena such as the annual change in densities of biological populations. It has been found that differential equation models with state variables depending continuously on time may too exhibit chaotic behavior. The Lorenz equations, a "simple" model for the onset of turbulence in the context of meteorological problems, is a wellknown example of such a system, see [17, 21]. It is understood that this system contains a so-called strange attractor.

In electronic circuits it has been observed that periodically forced nonlinear oscillators may behave chaotically in certain parameter ranges $[4$, 16]. In this paper we carry out an asymptotic

\footnotetext{
*Presently, Dept. of Applied Mathematics, Techn. Univ. Twente, The Netherlands.

** Presently, Nat. Inst. for Public Health and Environ. Protection, Leidschendam, The Netherlands.
}

analysis of such a system, the forced Van der Pol oscillator,

$$
\begin{aligned}
& \frac{\mathrm{d}^{2} x}{\mathrm{~d} t^{2}}+\nu\left(x^{2}-1\right) \frac{\mathrm{d} x}{\mathrm{~d} t}+x=\nu b(\nu) k \cos k t, \\
& b(\nu)=\alpha+\beta / \nu, \quad 0<\alpha<2 / 3,
\end{aligned}
$$

for large values of $\nu$. Eventually, we will construct an interval mapping that contains all necessary quantitative and qualitative information for describing the possible solutions of this system. Besides the well-known stable solutions of period $T=2 \pi(2 n-1) / k$, also irregular types of solutions are analyzed. Existence of such solutions was expected by Littlewood [15] and made plausible by Levinson [13] in a study of a related piece-wise linear equation. The horse shoe mapping created by Smale $[23,24]$ turned out to be an important tool in establishing the existence of these irregular solutions. Levi [11, 12] used this concept and symbolic dynamics (see also Guckenheimer [10]) in his study of a modified version of (1.1) which comes close to the piece-wise linear variant of Levinson. Our results agree qualitatively with those of Levi. Furthermore, (1.1) has been solved 
numerically by Flaherty and Hoppensteadt [3]. A comparison shows that there is also a good agreement between the outcome of their work and that of our asymptotic investigation.

In section 2 a qualitative description of the solutions of (1.1) is given. Most of it is based on the work of Levi. In section 3 we derive matched local asymptotic solutions for the regular subharmonic solutions of (1.1). Furthermore, we construct domains $\Omega_{n}$ in the $b, \nu$-plane, where the conditions for the existence of a formal asymptotic solution of period $T=2 \pi(2 n-1) / k$ are satisfied. In section 4 the same asymptotic method is used for analyzing irregular solutions, which, in Littlewood's terminology, exhibit dips and slices. We also construct the interval mapping $P$. There exists an equivalence relation between the solutions of (1.1) and the iterates of the mapping $P$. In section 5 the properties of $P$ are analyzed in detail. Using symbolic dynamics we are in the position to handle also the irregular solutions. Finally, in section 5.4 we numerically trace a stable irregular periodic solution.

\section{The annulus mapping and symbolic dynamics}

\subsection{Properties of the Poincare mapping}

Following Levi [11], we consider (1.1) in the form of a system of two first order differential equations

$\dot{x}=\nu\left(y-1 / 3 x^{3}+x\right)$,

$\dot{y}=-x / \nu+b k \cos k t$,

and introduce the Poincaré mapping $D$ :

$D(x(0), y(0))=(x(2 \pi / k), y(2 \pi / k))$.

It can be shown that a properly chosen annulus $\mathrm{A}$ in the $x, y$-plane is mapped into an annulus $\mathrm{A}^{\prime}$ under $D^{m}$ for $m$ sufficiently large with $\mathrm{A}^{\prime}$ having a thickness $\mathcal{O}\left(\exp \left(-c \nu^{2}\right)\right)$ near the branches of $y=$ $1 / 3 x^{3}-x$ and of order $\mathcal{O}\left(\nu^{-1 / 2}\right)$ at the horizontal parts where it jumps from one branch to the other, see fig. 1. Taking iterates at times $t=\phi+2 \pi j / k$, $j=1,2, \ldots$ we find an annulus shifted downwards. At $\phi=\pi / k$ this shift is at its maximum. Then the annulus $\mathrm{A}^{\prime}$ is found from the present one by reflection with respect to the origin.

Within the annulus $\mathrm{A}^{\prime}$ one may cut out a small part $\mathrm{R}$ such that for all $s=(x, y) \in \mathrm{A}, D^{j_{s}} \in \mathrm{R}$ for certain $j \in \mathbb{N}$. Note that there is even an infinite sequence $j_{k}, k=1,2, \ldots$ with $D^{j_{k}} \in \mathrm{R}$. Therefore it is sufficient to study the mapping $\hat{P}: \mathrm{R} \rightarrow \mathrm{R}$, where $\hat{P}_{S}=D^{j_{S}}$ with $j$ being the first integer for which $D^{j_{s}} \in \mathrm{R}$. By taking $D(s)=s$ for $s \in \mathrm{R}$ we obtain a continuous mapping $\hat{P}$ on R. Identification of the upper and lower sides of $\mathrm{R}$ makes $\mathrm{R}$ an annulus. Note that no points in $\mathrm{R}$ correspond with each other under $\hat{P}$. Thus, the study of the Poincare mapping $D$ is reduced to the analysis of the annulus mapping $\hat{P}: \overline{\mathrm{R}} \rightarrow \overline{\mathrm{R}}$. Because of the symmetry with respect to the origin there is a same area $\mathrm{R}^{\prime}$ in the opposite quadrant of the $x, y$-plane. Clearly, it is sufficient to study the mapping $P: \overline{\mathrm{R}}$ $\rightarrow \overline{\mathrm{R}}^{\prime}$ as $\hat{P}=P \circ P$.
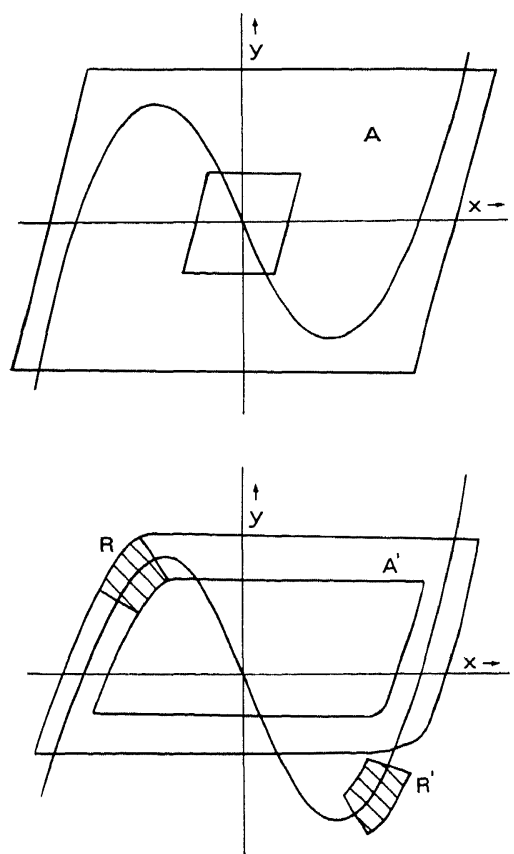

Fig. 1. Contraction of an annulus by repeated mappings $D$. 


\subsection{A circle mapping}

In a first approach we ignore the thickness of $\mathrm{R}$ and interpret $P$ as a circle mapping from $S^{1}$ to $S^{1}$. Graphically, $P$ behaves qualitatively as follows (see fig. 2). A small arc $\Delta$ of order $O\left(\nu^{-1}\right)$ of $S^{1}$ is stretched by $P$ to, say, 1.5 times the length of $\mathrm{S}^{1}$, while the remaining part $S^{1} \backslash \Delta$ is deformed simply by closing the image of $\Delta$. As we will find out, an increase of the forcing $b$ means a clockwise rotation of the image $P(\Delta)$. This immediately leads to the presentation in fig. 3, where for two different values of $\mathrm{b}$ we have given the graph of $P$. The two cases $\tilde{\mathrm{A}}$ and $\tilde{\mathrm{B}}$ correspond to mappings $P$ with quite different properties. Levi [11] makes these differences visible in the associated graphs of the annulus mapping $P$ with $\mathrm{R}$ being essentially an annulus and not a circle.

\subsection{The annulus mapping}

In fig. $4 \mathrm{a}$ we depicted the simple configuration of one stable fixed point $\mathrm{p}$ and an unstable saddle point $\mathrm{z}$ for the mapping $P$. By rotation we arrive

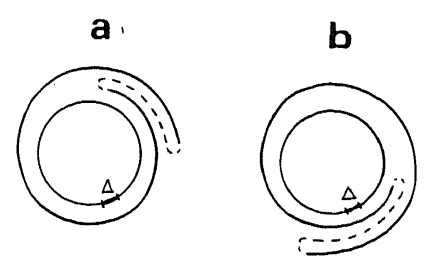

Fig. 2. Circle mapping. a) Case $\tilde{A} ; b)$ case $\tilde{B}$.

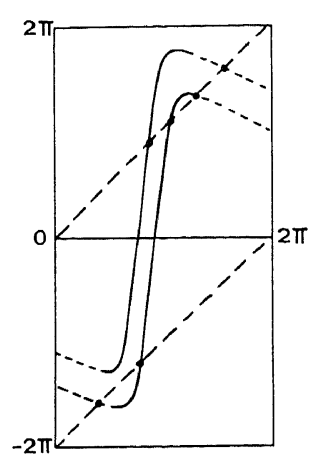

Fig. 3. Graphs of the two cases. at the more complicated configuration of fig. $4 \mathrm{~b}$ : it has two stable fixed points $\mathrm{p}_{1}, \mathrm{p}_{2}$ and two unstable saddle point type of fixed points $z_{1}$ and $z_{2}$. There is still another set of points $C$, which cannot decide to which basin of attractors they belong. $\mathrm{C}$ is a Cantor set of measure zero, which is invariant under $P$. In fact $C$ contains a nontrivial "attractor" of the type known as the horseshoe, see Smale [23, 24]. Symbolic dynamics (see e.g. [22]) gives a description of $P$ restricted to C, see Moser [19]. We set

$\Delta \cap P(\Delta)=\left\{\mathrm{H}_{0}, \mathrm{H}_{1}, \mathrm{H}_{3}\right\}$

see fig. $4 b$, and define $V_{0}, V_{1}$ and $V_{3}$ by

$P\left(\mathrm{~V}_{i}\right)=\mathrm{H}_{i}, \quad i=0,1,3$

Furthermore, let $\mathrm{H}_{2}=\mathrm{V}_{0} \cap P(\Delta)$, then $\mathrm{V}_{2}$ is defined by

$P\left(\mathrm{~V}_{2}\right)=\mathrm{H}_{2}$.

We now introduce the transition matrix $M$ by

$M_{i j}=0, \quad$ if $\mathrm{V}_{i} \cap \mathrm{H}_{j}=\varnothing$

and

$M_{i j}=1$ otherwise,

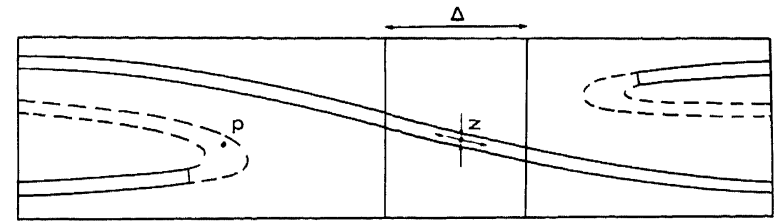

a

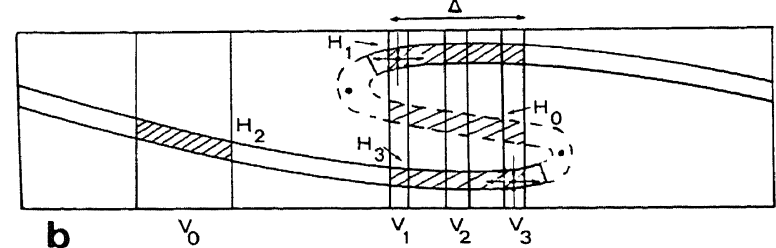

Fig. 4. The annulus mapping. (a) Case $\tilde{\mathrm{A}}$; (b) case $\tilde{\mathrm{B}}$. 
so

$M=\left(\begin{array}{llll}0 & 1 & 1 & 1 \\ 0 & 1 & 1 & 1 \\ 1 & 0 & 0 & 0 \\ 0 & 1 & 1 & 1\end{array}\right)$

\subsection{The use of symbolic dynamics}

The basic idea of symbolic dynamics is to introduce a space $\Sigma$ of all possible biinfinite strings of a given set of symbols. Referring to the indices of $\mathrm{V}_{i}$ and $\mathrm{H}_{i}$ of section 2.3 we take the symbols $0,1,2$ and 3:

$\Sigma=\{0,1,2,3\}^{\mathbf{Z}}$

Thus, an element $\boldsymbol{a}=\left(\ldots, a_{-1}, a_{0}, a_{1}, \ldots\right) \in \Sigma$ may read

$$
\ldots 01121322110 \ldots
$$

By posing restrictions upon the type of symbol that follows a given symbol one introduces a subspace of $\Sigma$ :

$\Sigma_{M}=\left\{a \in \Sigma \mid M_{a_{i} a_{i+1}}=1\right\}$

Let $M$ be given by (2.4). The mapping $P: \mathrm{C} \rightarrow \mathrm{C}$ is topologically conjugate with the shift $\sigma: \Sigma_{M} \rightarrow$ $\Sigma_{M}$, where $\sigma$ satisfies

$$
[\sigma(\boldsymbol{a})]_{i}=a_{i+1}, \quad i \in \mathbb{Z}
$$

Thus, there is a one to one corresponding $\theta$ between $C$ and $\Sigma_{M}$ :

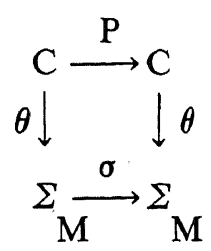

\subsection{Some remarks about the annulus mapping}

Returning to the annulus (or circle) mapping we observe the following dependence of this mapping upon $b$. There is a subdivision of the $b$-interval $(0,2 / 3)$ into subintervals $\tilde{\mathrm{A}}_{k}$ and $\tilde{\mathrm{B}}_{k}$ separated by small intervals $\tilde{\mathrm{g}}_{k}$, such that for $b \in \tilde{\mathrm{A}}_{k} P$ acts as given in fig. $4 \mathrm{a}$ (or fig. $2 \mathrm{a}$ ), while for $b \in \tilde{\mathrm{B}}_{k}$ the behavior can be understood from fig. $4 \mathrm{~b}$ (or fig. $2 b)$. In summary we conclude that for $b \in \tilde{\mathrm{A}}_{k}$ there is only one stable solution and except for one saddle point all solutions tend to this stable one. For $b \in \tilde{\mathrm{B}}_{k}$ two stable solutions exist. Except for a Cantor set of measure zero all solutions tend to one of the attractors.

As $b$ crosses a separation interval $\tilde{g}_{k}$ there is a sequence of bifurcations and the remarkable phenomenon occurs that for uncountably many $b \in \tilde{\mathrm{g}}_{k}$ there exist infinitely many stable fixed points of $P$ or of its iterates (see e.g. Newhouse and Palis [20]). In trying to understand this complicated bifurcation pattern we need more information about the mapping $P$. Intuitively, it is felt that these bifurcations are related with the disappearance of intersections $\mathrm{V}_{i} \cap \mathrm{H}_{j}$, see fig. $4 \mathrm{~b}$, and that therefore certain finite sequences in $\Sigma_{M}$ are forbidden.

It is obvious that an exact description of the mapping $P$ on the interval as sketched in fig. 3 is the first step in the full understanding of the bifurcation pattern. It is our goal to give a complete description of this mapping and its dependence upon the parameters. In the following sections we will construct matched local asymptotic solutions of (1.1), which eventually lead to a mapping on an interval with the same properties as $P$.

Finally, it is noted that the study of interval mappings has become a field of growing importance in the analysis of dynamical systems, see e.g. [20]. Various bifurcation problems have been solved by using the concept of mapping on an interval, but there still remain many questions about the exact description of families of interval mappings. In particular we mention the chaotic behavior of mappings within certain parameter ranges. 


\section{Matched local asymptotic solutions}

\subsection{Outline of the method}

A solution of (1.1) has a behavior that is characteristic for singular perturbation problems. Locally, the solution exhibits a boundary layer type of action like one meets in problems of fluid mechanics. On the other hand it passes a large time interval, where a two time scales expansion can be applied. Finally, we distinguish a sequence of points, determined by the intersections with the lines $x= \pm 1$, where the local behavior of the solutions is analyzed by a stretching procedure in both the dependent and independent variable. For a complete picture of the different regions which are successively crossed by the solution we refer to fig. 5. The regions $\mathrm{A}_{m}, \mathrm{~B}_{m}, \mathrm{C}$ and $\mathrm{A}$ indicated in this figure will be intrinsically defined by the local asymptotic behaviour of the solution of (1.1). The method of matched asymptotic expansions (see e.g. [1]) yields formal local asymptotic solutions in which the integration constants are determined by averaging conditions and by matching pairs of local solutions of adjacent regions. These computations have been carried out in $[6,7]$. In the next sections we summarize the results. It is emphasized that this approach is formal and that, for the type of problem we are dealing with, there is no proof of correctness that justifies this approach. However, the present method results in a clear qualitative picture of the solution and, in addition, provides us with quantitative information about the existence of subharmonic and other solutions. Comparing this outcome with analytical and numerical results for the same or related problems $[2,11]$, we observe a good agreement. Furthermore, matching methods require a high degree of internal consistency. That is: two neighboring local solutions match, if they exhibit the same behaviour in a relatively broad domain of overlap. Thus, there always are checks on the correctness of the solutions. This type of consistency we also meet in the final result: we analyse here the case $0<\alpha<$ $2 / 3$ and derive conditions for $\beta$ in order to have a

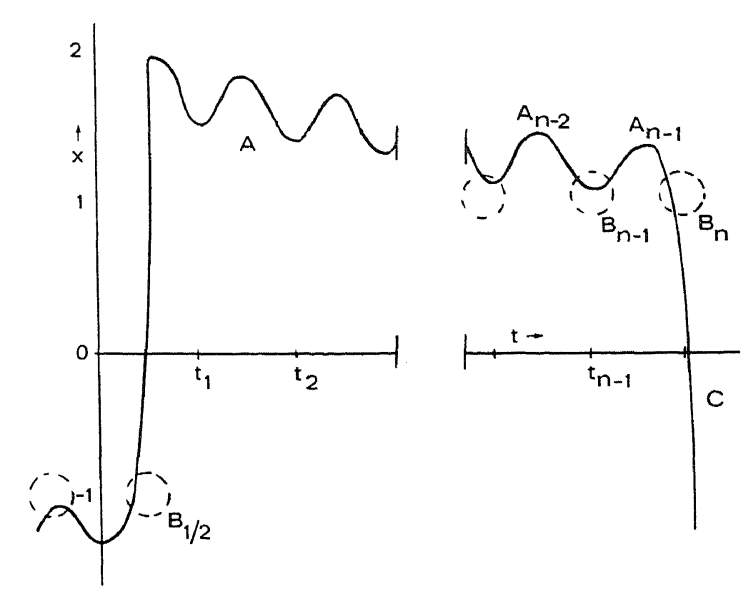

Fig. 5. Regions for local asymptotic solutions.

subharmonic of order $n$. In the limit $\alpha \rightarrow 0$ and $\alpha \rightarrow 2 / 3$ these conditions need to match the ones obtained from studying the special cases $\alpha=0$ and $\alpha=2 / 3$, see [5,8]. As will be seen this turns out to be correct indeed.

\subsection{The regions $\mathrm{A}_{m}$ and $\mathrm{B}_{m}$}

A region $\mathrm{A}_{m}$, where the solution has a typical local asymptotic behaviour, is defined as follows.

Definition 3.1. A solution of (1.1) is said to be in region $\mathrm{A}_{m}$ of the $x, t$-plane if for

$t_{m}+L \nu^{-1 / 2}<t<t_{m+1}-L \nu^{-1 / 2}$,

$t_{m}=(-\pi / 2+2 \pi m) / k$,

with $L$ arbitrarily large (but independent of $\nu$ ) the inequality $|x(t)|>1$ holds, and if, moreover

$\left|x\left(t_{m}+L \nu^{-1 / 2}\right)\right|=1+\mathcal{O}\left(\nu^{-1 / 2}\right)$

and

$\left|x\left(t_{m+1}-L \nu^{-1 / 2}\right)\right|=1+\mathcal{O}\left(\nu^{-1 / 2}\right)$.

Let us first analyse the local asymptotic solution for a region $\mathrm{A}_{m}$ above the $t$-axis with $m$ a positive integer, see fig. 5. It is assumed that the solution 
can be expanded as

$x(t, \nu)=x_{m 0}(t)+\nu^{-1} x_{m 1}(t)+\cdots$.

Substitution in (1.1) yields after grouping of terms of order $\mathcal{O}(v)$ and $\mathcal{O}(1)$ the following equations for $x_{m i}, i=1,2$,

$\left(x_{m 0}^{2}-1\right) \mathrm{d} x_{m 0} / \mathrm{d} t=\alpha k \cos k t$,

$$
\begin{gathered}
\left(x_{m 0}^{2}-1\right) \frac{\mathrm{d} x_{m 1}}{\mathrm{~d} t}+2 x_{m 0} \frac{\mathrm{d} x_{m 0}}{\mathrm{~d} t} x_{m 1} \\
=-\frac{\mathrm{d}^{2} x_{m 0}}{\mathrm{~d} t^{2}}-x_{m 0}+\beta k \cos k t,
\end{gathered}
$$

or after integration

$$
\begin{aligned}
1 / 3 x_{m 0}^{3}-x_{m 0}= & \alpha \sin k t+C_{0}^{(m)} \\
\left(x_{m 0}^{2}-1\right) x_{m 1}= & -\frac{\mathrm{d} x_{m 0}}{\mathrm{~d} t}-\int_{t_{m-1}}^{t} x_{m 0}(\bar{t}) \mathrm{d} \bar{t} \\
& +\beta \sin k t+C_{1}^{(m)}
\end{aligned}
$$

Since for $t \uparrow t_{m} x$ approaches the value 1, we have $C_{0}^{(m)}=\alpha-2 / 3$ and asymptotically

$$
x=1-\frac{1}{2} a^{2}\left(t-t_{m}\right)+K_{m}\left\{a^{2}\left(t-t_{m}\right) \nu\right\}^{-1}
$$

with

$$
\begin{gathered}
K_{m}=-\frac{1}{2} a^{2}+\left(-C_{1}^{(m)}+\beta+2 \pi I\right), \quad a=\sqrt[4]{2 \alpha k^{2}}, \\
I=\frac{1}{2 \pi} \int_{t_{m-1}}^{t_{m}} x_{m 0}(t) \mathrm{d} t, \\
x_{m 0}(t)=2 \cos \left[\frac{1}{3} \arccos \left\{\frac{3}{2} \alpha \sin k t+\frac{3}{2} \alpha-1\right\}\right. \\
\left.+\frac{2}{3} \pi j\right],
\end{gathered}
$$

where $j=0$.

Next we deal with the region $\mathrm{B}_{m}$.

Definition 3.2. The solution of (1.1) is said to be region $\mathrm{B}_{m}$, if for

$$
\begin{aligned}
& t_{m}-L \nu^{-1 / 2}<t<t_{m}+L \nu^{-1 / 2}, \\
& t_{m}=(-\pi / 2+2 \pi m) / k,
\end{aligned}
$$

with $L$ arbitrary there exists a constant $M$ such that ||$x(t)|-1|<M \nu^{-1 / 2}$.

From (3.6) we conclude that one may expect a different asymptotic behaviour of the solution in a $\nu^{-1 / 2}$-neighborhood of $(x, t)=\left(1, t_{m}\right)$. We, therefore, introduce the local variable $\xi=\left(t-t_{m}\right) \nu^{1 / 2}$ and expand $x$ as follows:

$x=1+\nu^{-1 / 2} V_{m 0}(\xi)+\nu^{-1} V_{m 1}(\xi)+\cdots$.

Substitution in (1.1) yields for the leading terms of $\mathcal{O}\left(\nu^{1 / 2}\right)$

$\frac{\mathrm{d}^{2} V_{m 0}}{\mathrm{~d} \xi^{2}}+2 V_{m 0} \frac{\mathrm{d} V_{m 0}}{\mathrm{~d} \xi}=\alpha k^{2} \xi$

Furthermore, $V_{m 0}$ should match (3.6) or

$V_{m 0}(\xi) \simeq-\frac{1}{2} a^{2} \xi+K_{m}\left(a^{2} \xi\right)^{-1}, \quad$ as $\xi \rightarrow-\infty$.

After carrying out a transformation of type $V_{m 0}=$ $Z^{\prime} / Z$, we find the following solution for (3.10) and (3.11):

$$
V_{m 0}(\xi)=-a D_{K_{m} / a^{2}}^{\prime}(-a \xi) / D_{K_{m} / a^{2}}(-a \xi),
$$

where $D_{p}(x)$ denotes the parabolic cylinder function of order $p$.

Let us assume that $K_{m}$ is negative, then the parabolic cylinder function $D_{K_{m} / a^{2}}$ has no zeroes and $V_{m 0}$ remains regular. The asymptotic solution will lose its validity for $\xi \rightarrow \infty$, as

$$
V_{m 1}(\xi) \simeq \frac{1}{2} a^{2} \xi-\left(K_{m} / a^{2}+1\right) \xi^{-1} .
$$

Now the solution enters the region $\mathrm{A}_{m+1}$, where (3.1)-(3.8) hold with $m$ replaced by $m+1$. For 
$t \downarrow t_{m}$, this solution behaves as

$$
\begin{aligned}
x_{m+1}(t) \simeq & 1+\frac{1}{2} a^{2}\left(t-t_{m}\right) \\
& -K_{m+1}\left\{a^{2}\left(t-t_{m}\right) \nu\right\}^{-1}
\end{aligned}
$$

Consequently, the matching conditions result in the recursive relation

$K_{m+1}=Q\left(K_{m}\right) \equiv K_{m}+2 \pi I$

Similar matched local solutions are found for the regions $\mathrm{A}_{m}$ and $\mathrm{B}_{m}$ below the $x$-axis with $m=1 / 2,-1 / 2,-3 / 2, \ldots$. For such regions $\mathrm{A}_{m}$ (3.8b) holds with $j=1$. Clearly, there exists a value of $m$ for which $0<K_{m}<2 \pi I$. Without loss of generality this may be for $m=1 / 2$ below the $t$-axis, see fig. 5 . The same occurs above the $t$-axis the first time for $m=n \geq 1$ with $n$ integer.

For $K_{n}>0$ the parabolic cylinder function has one or more zeros. Let $\xi_{0}$ be the first zero one meets coming from $-\infty$. For $\xi \uparrow \xi_{0}$ we have

$$
\begin{aligned}
V_{n}(\xi) \simeq & \left(\xi-\xi_{0}\right)^{-1} \\
& +\frac{1}{3} a^{2}\left(\frac{1}{4} a^{2} \xi_{0}^{2}-K_{n}-\frac{1}{2}\right)\left(\xi-\xi_{0}\right)
\end{aligned}
$$

and the solution leaves there the $\nu^{-1 / 2}$-neighborhood of $(x, t)=\left(1, t_{n}\right)$. A similar behaviour is found in the region $B_{1 / 2}$.

There is a strong similarity between the mapping $Q(K)$ of (3.15) and $D(x, y)$ of (2.2). As explained in section 2.1 Levi derived from $D$ a mapping $P: \mathrm{R} \rightarrow \mathrm{R}^{\prime}$, where $\mathrm{R}$ is a strip in the phase plane and $R^{\prime}$ its reflection with respect to the origin. Likewise we will construct a mapping $P(K)$ of this interval $[0,2 \pi I]$ into itself. Starting in region $\mathrm{B}_{1 / 2}$ with $K_{1 / 2}$ within this interval we look for the smallest integer $n \geq 1$ such that $K_{n}$ returns in this interval, then $P\left(K_{1 / 2}\right)=K_{n}$. In order to derive an explicit expression for $P(K)$ we have to construct matched local solutions for the remaining regions $\mathrm{C}$ and $\mathrm{A}$.

\subsection{The region $\mathrm{C}$}

At $t=t_{1 / 2}+\xi_{0} \nu^{-1 / 2}$ the solution leaves the $\mathrm{B}_{1 / 2}$-region and enters the boundary layer region $\mathrm{C}$.

Definition 3.3. If for some $t=t_{c}$ the solution of (1.1) satisfies $x\left(t_{c}\right)=0$, then it is said to be in region $\mathrm{C}$ for $t_{c}-L \nu^{-1}<t<t_{c}+L \nu^{-1}$ with $L$ arbitrary (but independent of $\nu$ ).

We introduce the local variable

$\eta=\left(t-t_{1 / 2}-\xi_{0} \nu^{-1 / 2}\right) \nu$

Assuming that the solution can be expanded as

$x(t, \nu)=W_{0}(\eta)+\nu^{-1} W_{1}(\eta)+\nu^{-2} W_{2}(\eta)+\cdots$,

we arrive at a recurrent system of equations for $W_{i}$,

$\frac{\mathrm{d}^{2} W_{0}}{\mathrm{~d} \eta^{2}}+\left(W_{0}^{2}-1\right) \frac{\mathrm{d} W_{0}}{\mathrm{~d} \eta}=0$,

$\frac{\mathrm{d}^{2} W_{1}}{\mathrm{~d} \eta^{2}}+\left(W_{0}^{2}-1\right) \frac{\mathrm{d} W_{1}}{\mathrm{~d} \eta}+2 W_{0} W_{1} \frac{\mathrm{d} W_{0}}{\mathrm{~d} \eta}=0, \ldots$

According to (3.16), the local solution (3.18) matches the asymptotic solution for region $B_{1 / 2}$, if for $\eta \rightarrow-\infty$

$$
\begin{aligned}
& W_{0}=-1-1 / \eta \\
& W_{1}=-\frac{1}{3} a^{2}\left(\frac{1}{4} a^{2} \xi_{0}^{2}-K_{1 / 2}-\frac{1}{2}\right) \eta
\end{aligned}
$$

Condition (3.21a) is satisfied by solutions of (3.19) of the form

$\frac{1}{-1-W_{0}}+\frac{1}{3} \ln \left\{\frac{W_{0}-2}{-1-W_{0}}\right\}=-\eta+$ constant 
while because of $(3.21 \mathrm{~b})$ the integrated equation (3.20) must have the form

$\frac{\mathrm{d} W_{1}}{\mathrm{~d} \eta}+\left(W_{0}^{2}-1\right) W_{1}=-a^{2}\left(\frac{1}{4} a^{2} \xi_{0}^{2}-K_{1 / 2}-\frac{1}{2}\right)$.

As $\eta \rightarrow \infty$ the boundary layer region is left at exponential rate

$$
\begin{aligned}
x= & 2-\frac{1}{3} a^{2}\left(\frac{1}{4} a^{2} \xi_{0}^{2}-K_{1 / 2}-\frac{1}{2}\right) \nu^{-1} \\
& +\mathcal{O}\left(\exp \left\{-3\left(t-t_{1 / 2}-\xi_{0} \nu^{-1 / 2}\right) \nu\right\}\right) .
\end{aligned}
$$

\subsection{The two variable expansion for region A}

In the region $\mathrm{A}$ the solution has an oscillatory behaviour in the $\mathcal{O}(1)$ time scale and its average value decreases slowly in the $\mathcal{O}(\nu)$ scale.

Definition 3.4. Let at time $t=t^{*}$ the solution of (1.1) satisfy $\left|x\left(t^{*}\right)\right|=2+\mathcal{O}\left(\nu^{-1}\right)$ then $x(t)$ is said to be in region A for $t \geq t^{*}$ until $|x(t)| \leq 1+$ $L \nu^{-1 / 2}$ for $L$ arbitrarily large but independent of $\nu$.

Using the two variable expansion procedure, as explained in Cole [1], we write the solution for the region $\mathrm{A}$ above the $t$-axis as

$x=x_{0}(t, \tau)+\nu^{-1} x_{1}(t, \tau)+\nu^{-2} x_{2}(t, \tau)+\cdots$,

with $\tau=\left(t-t_{1 / 2}\right) / \nu$. The coefficients satisfy a recurrent system of differential equations. The first one is

$$
\left(x_{0}^{2}-1\right) \mathrm{d} x_{0} / \mathrm{d} t=\alpha k \cos k t
$$

or

$$
\frac{1}{3} x_{0}^{3}-x_{0}=\alpha \sin k t+C_{0}(\tau),
$$

with a solution above the line $x=1$

$$
x_{0}(t, \tau)=2 \cos \left[\frac{1}{3} \arccos \left\{\frac{3}{2} \alpha \sin k t+\frac{3}{2} C_{0}(\tau)\right\}\right] \text {. }
$$

For the second equation we obtain

$$
\begin{aligned}
& \frac{\partial^{2} x_{0}}{\partial t^{2}}+\left(x_{0}^{2}-1\right)\left(\frac{\partial x_{0}}{\partial \tau}+\frac{\partial x_{1}}{\partial t}\right)+2 x_{0} x_{1} \frac{\partial x_{0}}{\partial t}+x_{0} \\
& =\beta k \cos k t
\end{aligned}
$$

or

$$
\begin{aligned}
\left(x_{0}^{2}-1\right) x_{1}= & -\frac{\partial x_{0}}{\partial t}-\int_{t_{1 / 2}}^{t} G_{0}(\bar{t}, \tau) \mathrm{d} \bar{t} \\
& +\beta \sin k t+C_{1}(\tau),
\end{aligned}
$$

with

$G_{0}(t, \tau)=x_{0}(t, \tau)+\partial C_{0} / \partial \tau$.

In order to remove secular terms in $x_{1}$ we set the following averaging condition:

$\int_{\tau \nu}^{t \nu+2 \pi / k} G_{0}(t, \tau) \mathrm{d} t=0$

or

$\frac{\partial C_{0}}{\partial \tau}=\frac{-k}{2 \pi} \int_{\tau \nu}^{\tau \nu+2 \pi / k} x_{0}(t, \tau) \mathrm{d} t$.

Since at time $t=t_{1 / 2}$ the solution starts at the value $x=2, C_{0}(0)$ takes the value $2 / 3-\alpha$. It will leave the region $A$ at a time $t_{m}=(-\pi / 2+2 \pi m) / k$ as then the line $x=1$ is approached. This takes place as $C_{0}(\tau)$ reaches the value $-2 / 3+\alpha$. From (3.33) it follows that in the slow time scale this will be for

$$
\Upsilon(\alpha)=\frac{2 \pi}{k} \int_{-2 / 3+\alpha}^{2 / 3-\alpha}\left\{\int_{0}^{2 \pi / k} x_{0}\left(t ; C_{0}\right) \mathrm{d} t\right\}^{-1} \mathrm{~d} C_{0} .
$$


It is easily verified that indeed $\Upsilon(0)=3 / 2-\ln 2$ and $\Upsilon(2 / 3)=0$. Finally, we derive from the averaging condition for $x_{2}(t, \tau)$ and explicitly solvable equation for $C_{1}(\tau)$. Substituting $t=t_{1 / 2}+\xi_{0} \nu^{-1 / 2}$ in (3.25) we obtain

$$
C_{1}(0)=C_{10}=\beta+a^{2}\left(K_{1 / 2}+\frac{1}{2}\right) .
$$

The equation for $C_{1}$ yields

$$
C_{1}(\Upsilon)=q(\alpha)\left\{C_{10}-\beta p(\alpha)\right\}, \quad \Upsilon=\Upsilon(\alpha),
$$

$p(\alpha)=\frac{k}{2 \pi} \int_{0}^{\Upsilon} \exp \left\{\frac{k}{2 \pi} \int_{0}^{\tau} \int_{\bar{\tau} \nu}^{\bar{\tau} \nu+2 \pi / k} \frac{1}{x_{0}^{2}-1} \mathrm{~d} t \mathrm{~d} \bar{\tau}\right\}$

$$
\times \int_{\tau \nu}^{\tau \nu+2 \pi / k} \frac{\sin k t}{x_{0}^{2}-1} \mathrm{~d} t \mathrm{~d} \tau
$$

$q(\alpha)=\exp \left\{\frac{-k}{2 \pi} \int_{0}^{\Upsilon} \int_{\tau \nu}^{\tau \nu+2 \pi / k} \frac{1}{x_{0}^{2}-1} \mathrm{~d} t \mathrm{~d} \tau\right\}$.

When the solution approaches a $\nu^{-1 / 2}$-neighborhood of $(x, t)=\left(1, t_{m}\right)$ the expansion (3.25) behaves asymptotically as

$$
\begin{aligned}
x \simeq & 1-\frac{1}{2} a^{2}\left(t-t_{m}\right)+\left\{-\frac{1}{2} a^{2}+\beta-C_{1}(\tau)\right. \\
& \left.-C_{0}^{\prime}(\Upsilon)\left(t_{m}-t_{1 / 2}-\Upsilon \nu\right)\right\}\left\{a^{2}\left(t-t_{m}\right) \nu\right\}^{-1} .
\end{aligned}
$$

From (3.6) we conclude that the solution for region A matches the ones from regions $\mathrm{A}_{n-m}$ for $m$ and $n$ of order $\mathcal{O}(\nu)$ giving

$$
C_{1}^{(m)}-I=C_{1}(\Upsilon)+C_{0}^{\prime}(\Upsilon)\left(t-t_{1 / 2}-\Upsilon \nu\right)
$$

or as $I=-2 \pi C_{0}^{\prime}(\Upsilon) / k$,

$$
C_{1}^{(m)}=C_{1}(\Upsilon)-\left(m-\frac{3}{2}\right) I+(2 \pi / k)^{-1} I \Upsilon \nu .
$$
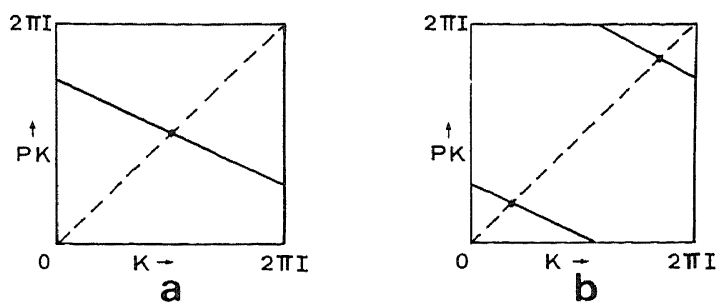

Fig. 6. The interval mapping $P(K)$. a) Case $\tilde{\mathrm{A}}_{n}$; b) case $\tilde{\mathrm{B}}_{n}$.

3.5. Symmetric solutions of period $T=2 \pi(2 n-$ 1) $/ k$

The construction of a symmetric periodic solution of period $T=2 \pi(2 n-1) / k$ with $x(t)=$ $-x(t-\pi(2 n-1) / k)$ is completed as follows. From the relations (3.7a), (3.15), (3.35), (3.36) and (3.8) we derive the mapping from $K_{1 / 2}$ with range $(0,2 \pi I)$ to $K_{n}$ within the same range: $K_{n}=$ $P\left(K_{1 / 2}\right)$. It turns out to be a simple linear or piece-wise linear relation, see fig. 6 ,

$$
\begin{aligned}
& P(K)=-q K+R(n(K)), \\
& R=(1+q+p q) \beta-\frac{1}{2} a^{2}(1+q)+2 \pi\left(n-\frac{1}{2}\right) I \\
& \quad-k I \Upsilon \nu,
\end{aligned}
$$

with $n$ such that $P(K)$ remains within the range $(0,2 \pi I)$. The functions $I(\alpha)$ and $\Upsilon(\alpha)$ are sketched in fig. 7. Fig. 8 depicts the auxiliary functions $p(\alpha)$

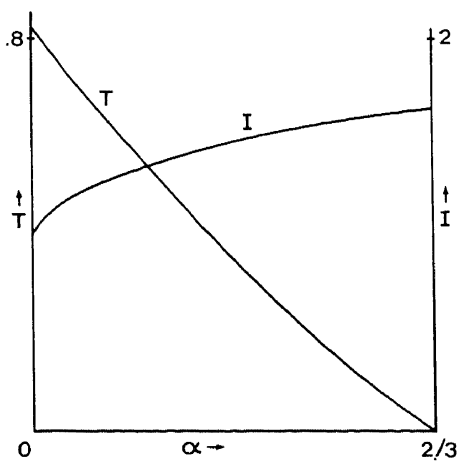

Fig. 7. The period $\Upsilon(\alpha)$ and characteristic function $I(\alpha)$. 


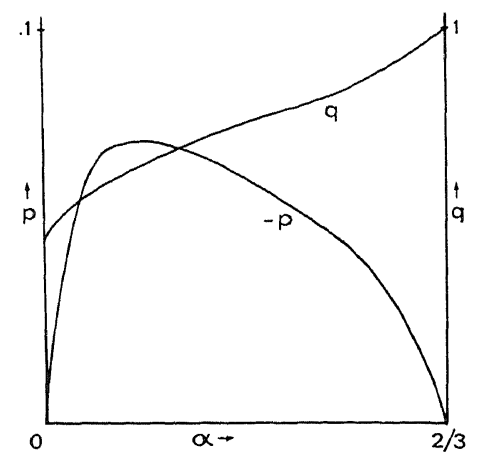

Fig. 8. The auxiliary functions $p(\alpha)$ and $q(\alpha)$.

and $q(\alpha)$. As $\beta$ increases the line $P(K)$ shifts upwards and as it reaches the upper boundary we lower $n$ by 1 . It is possible to compute the $\beta$-intervals $\left(\underline{\beta}_{n}, \bar{\beta}_{n}\right)$ where $P$ has a fixed point that corresponds with a solution of period $T=2 \pi(2 n-$
$1) / k$. These intervals overlap,

$$
\underline{\beta}_{n}<\bar{\beta}_{n+1}<\underline{\beta}_{n-1}<\bar{\beta}_{n} .
$$

Thus, there exist $\beta$-intervals with one fixed point (case $\tilde{\mathrm{A}}_{n}$ ) and with two fixed points (case $\tilde{\mathrm{B}}_{n}$ ). In fig. 9 we give the domain $\Omega_{n}$ in the $b, \nu$-plane with a solution of period $T=2 \pi(2 n-1)$. The following procedure has been carried out to find these domains for $k=1, n=1,2,3$ and 4. Step 1: a value of $\nu$ is fixed. Step 2: $\alpha$ is determined such that $\Upsilon(\alpha) \nu=2 \pi\left(n-\frac{1}{2}\right)$. Step 3: the $\beta$-interval is computed for which $P$ has a fixed point. Step 4: the line $\nu=$ constant is within the domain $\Omega_{n}$ for $b=\alpha+\beta / \nu$ with $\beta$ within the computed interval. The procedure is repeated for different values of $\nu$. The domains $\Omega_{n}$ agree quite well with the corresponding domains by Flaherty and Hoppensteadt [3]. For $b<1 / \nu$ we used the asymptotic solution of [8].

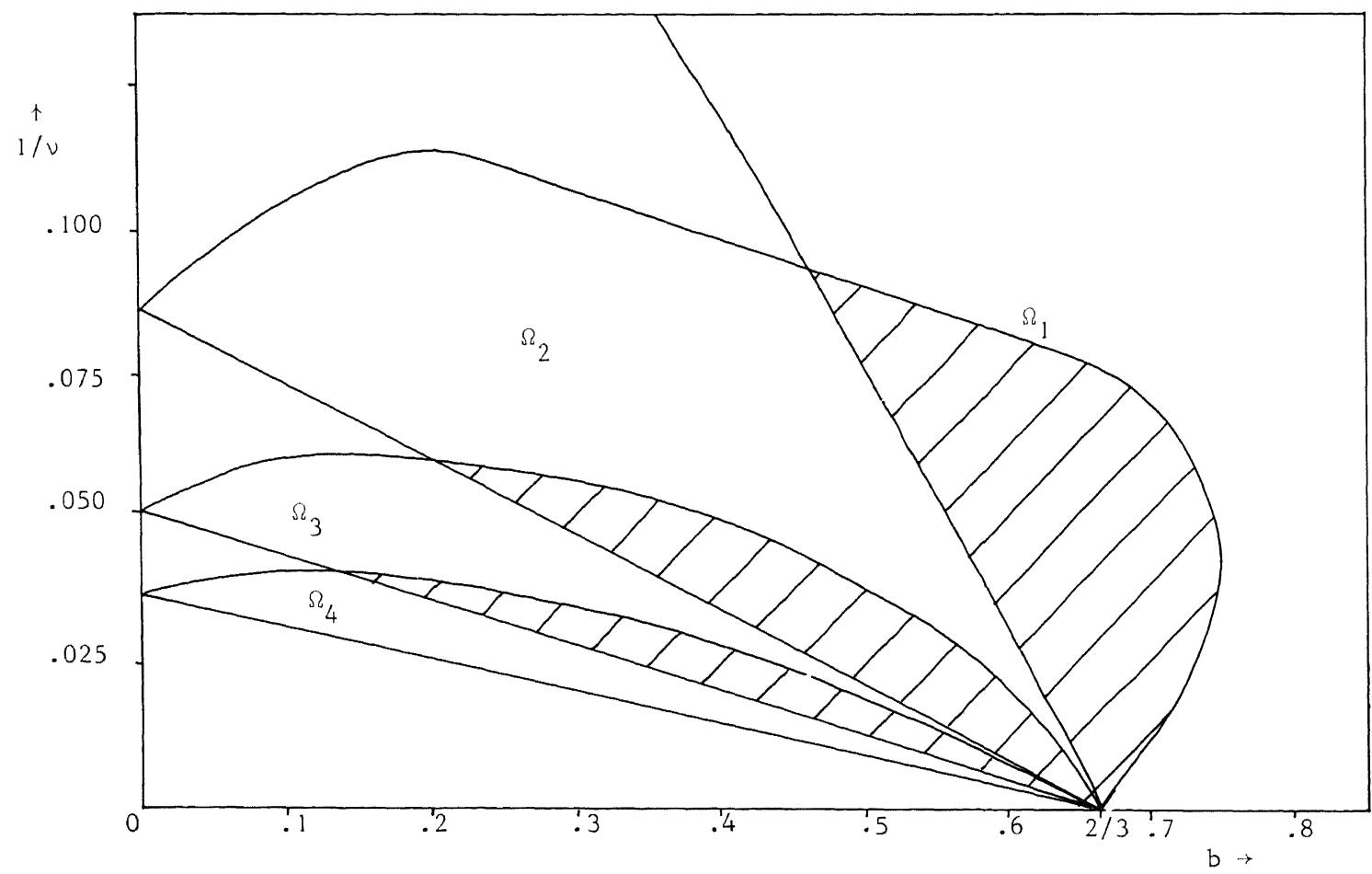

Fig. 9. The domains $\Omega_{n}$ in the parameter plane with subharmonics of period $T=2 \pi(2 n-1)$. 


\section{Irregular solutions: dips and slices}

\subsection{The additional local asymptotic solutions}

When $K_{1 / 2}$ or $K_{n}$ is near one of the end points of the interval $(0,2 \pi I)$ the solution will follow a completely different path before entering a type A region. In fig. 10 we explore this phenomenon by solving (1.1) numerically for two sets of starting values that agree up to the fifth decimal. Fig. 11 gives the regions, where the solution has its own local asymptotic behaviour. We consider the case where

$K_{1 / 2}=\sigma \exp (-d v)$

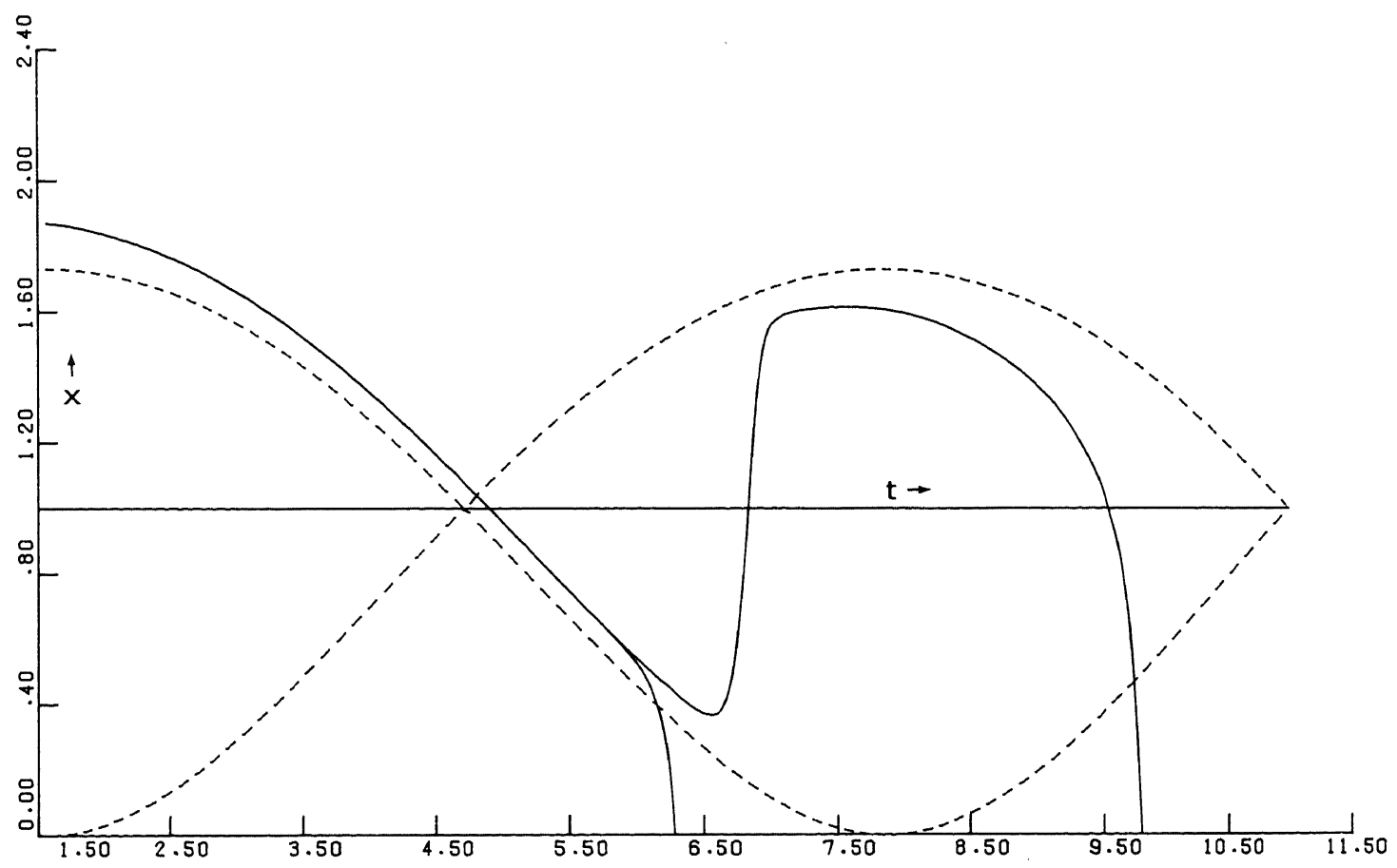

Fig. 10. Two numerical solutions for $\alpha=1 / 3, \beta=0, \nu=15$ and $x^{\prime}(\pi / 2)=-0.0521795, x(\pi / 2)=1.8711914(\operatorname{dip}), x(\pi / 2)=$ 1.8711901 (slice).
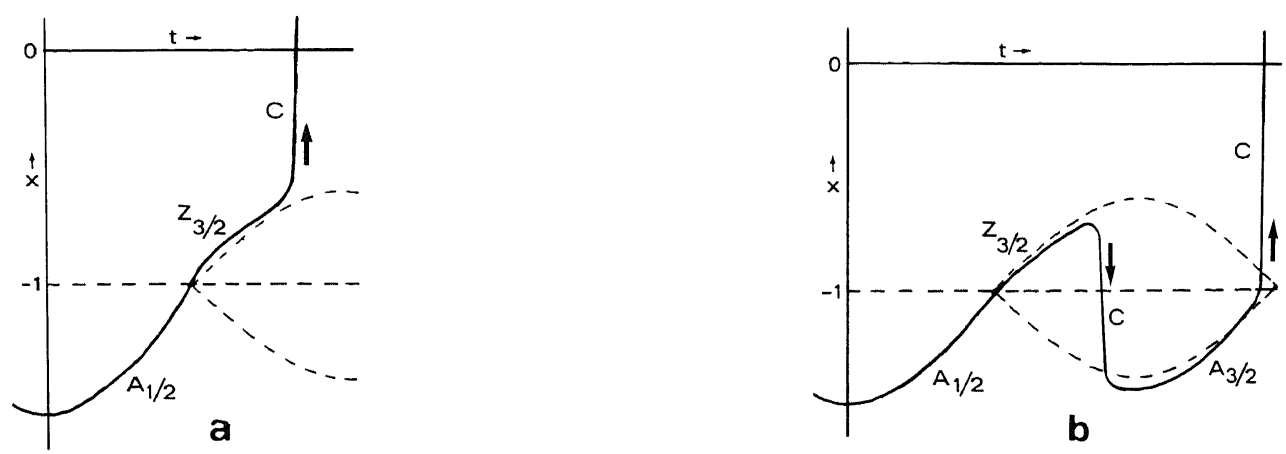

Fig. 11. Regions for local asymptotic solutions in the case of dips and slices. a) Slice; b) dip. 
local behaviour near the line $x=-1$, which we indicate by dips and slices of the solution, see fig. 11.

If for a moment we take $\sigma=0$, then the expansion (3.1) of regions $A_{1 / 2}$ remains regular and at $t=t_{1 / 2}$ the solution smoothly switches to a different expansion with leading term

$$
\begin{gathered}
x_{3 / 2}(t)=2 \cos \left[\frac{1}{3} \arccos \left(\frac{3}{2} \alpha \sin k t+\frac{3}{2} \alpha-1\right)\right. \\
\left.+\frac{4}{3} \pi\right]
\end{gathered}
$$

being the second branch of (3.4). This solution will hold asymptotically for the region $Z_{3 / 2}$ with $t>$ $t_{1 / 2}$.

We will deal with a regular asymptotic solution $\hat{x}(t ; \nu)$ of the form (3.1), which has two distinct representations: one for $t<t_{1 / 2}$ being (3.1)-(3.5) with $m=\frac{1}{2}$ and $C_{1}^{(1 / 2)}$ such that $K_{1 / 2}=0$, and another one for $t>t_{1 / 2}$ given by (3.1) with $m=$ $3 / 2$, see (4.2). The equation for $x_{3 / 2,1}(t)$ is of type (3.5) with

$C_{Z 1}^{(3 / 2)}=\beta-\frac{1}{2} a^{2}$.

This regular solution is perturbed as follows:

$x=\hat{x}(t ; \nu)+V(t ; \nu)$.

Substitution in (1.1) yields for the leading terms in $V$

$\frac{\mathrm{d}^{2} V}{\mathrm{~d} t^{2}}+\nu \frac{\mathrm{d}}{\mathrm{d} t}\left\{\left(\hat{x}^{2}-1\right) V\right\}=0$.

Using (3.6) we obtain the condition

$V \simeq \frac{\sigma}{a^{2} \xi} \nu^{1 / 2} \mathrm{e}^{-d \nu}, \quad$ for $\xi \rightarrow-\infty$,

which is satisfied by

$$
\begin{aligned}
& V=\sigma \exp \{-\nu(A(t)+d)\}\left[\sqrt{\pi /\left(2 a^{2} \nu\right)}\right. \\
& \left.-\int_{t_{1 / 2}}^{t} \exp \{\nu A(\bar{t})\} \mathrm{d} \bar{t}\right], \\
& A(t)=\int_{t_{1 / 2}}^{t}\left\{\hat{x}^{2}(\bar{t} ; 0)-1\right\} \mathrm{d} \bar{t} .
\end{aligned}
$$

This asymptotic solution breaks down as $t$ approaches $t^{*}$ satisfying

$A\left(t^{*}\right)=-d$.

Assuming that $t^{*}<t_{3 / 2}$ we have to introduce another boundary layer type of solution for the region $\mathrm{C}$ :

$x=W_{0}(\eta)+\nu^{-1} W_{1}(\eta)+\cdots, \quad \eta=\left(t-t^{*}\right) \nu$,

with $W_{0}$ satisfying

$$
\begin{aligned}
& \frac{\ln \left|W_{0}-z_{*}\right|}{z_{*}^{2}-1}+\frac{\ln \mid W_{0}-a_{*}}{a_{*}^{2}-1}+\frac{\ln \left|W_{0}-x_{*}\right|}{x_{*}^{2}-1} \\
& =-\eta+\frac{\ln \sqrt{\pi /\left(2 a^{2} \nu\right)}}{z_{*}^{2}-1}
\end{aligned}
$$

in which $z_{*}=\hat{x}\left(t^{*} ; 0\right)$ and $a_{*}$ and $x_{*}$ are the two other roots of the algebraic equation

$1 / 3 W_{0}^{3}-W_{0}=1 / 3 z_{*}^{3}-z_{*}$,

with $a_{*}<-1$ and $x_{*}>1$. For $W_{1}$ we have

$$
\begin{aligned}
& W_{1}^{\prime}+\left(W_{0}^{2}-1\right) W_{1}=\alpha \eta k \cos k t^{*} \\
& \quad-\int_{t_{1 / 2}}^{t^{*}} \hat{x}(t ; 0) \mathrm{d} t+\beta \sin k t^{*}-\beta+\frac{1}{2} a^{2} .
\end{aligned}
$$

Taking $\sigma=1$ we find that $W_{0} \rightarrow x_{*}$ as $\eta \rightarrow \infty$ and matches the solution of region $A$, see (3.25), if

$C_{10}=-\beta+\frac{1}{2} a^{2}-\int_{t_{1 / 2}}^{t^{*}} \hat{x}(t ; 0) \mathrm{d} t$.

For $\sigma=-1, W_{0}$ tends to the other stable root $a^{*}<-1$ and matches the asymptotic solution for region $\mathrm{A}_{3 / 2}$ given by $x=\hat{a}(t ; \nu)$ satisfying (3.1)-(3.8) with $j=1, C_{0}^{(3 / 2)}=-\alpha+3 / 2$ and

$C_{1}^{(3 / 2)}=C_{Z 1}^{(3 / 2)}+\int_{t_{1 / 2}}^{t^{*}} \hat{x}(t ; 0) \mathrm{d} t$. 

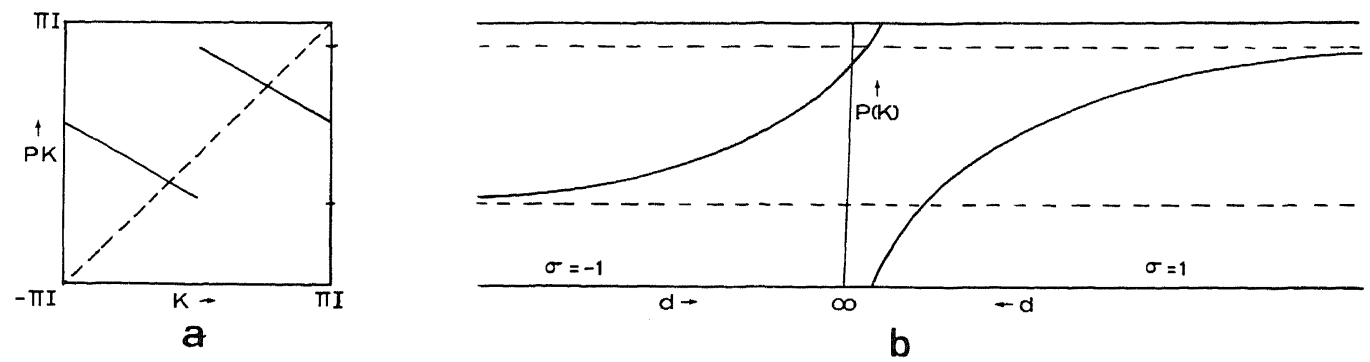

Fig. 12. a) The discontinuous (outer) expression for $P(K)$; b) the local (inner) expression for $P(K)$ with $K=\sigma \exp (-\nu d)$.

In the $B_{3 / 2}$-region a solution of type (3.9)-(3.14) holds with

$K_{3 / 2}=-\int_{t_{1 / 2}}^{t^{*}} \hat{x}(t ; 0) \mathrm{d} t-\int_{t^{*}}^{t_{3 / 2}} \hat{a}(t ; 0) \mathrm{d} t$.

After crossing the unstable interval $|x|<1$, the system arrives at the region $\mathrm{A}$, where it matches (3.25) because of (3.35) with $K_{1 / 2}$ replaced by $K_{3 / 2}$. In [7] the case where $d$ is sufficiently large (including $\sigma=0$ ) has been studied in the $\mathrm{B}_{3 / 2^{-}}$ region. We do not present this result here as it does not affect the matching relations given above.

\subsection{The interval mapping $P(K)$}

In order to analyse the mapping $P(K)$ with $K=\mathcal{O}(\exp (\nu))$ we shift the interval to $(-\pi I, \pi I)$ so that $K=0$ becomes an internal point. Bringing all matching relations together, we express $K_{n}$ in $K_{1 / 2}$ for $K_{1 / 2}= \pm \exp (-d \nu)$ with $d=\mathcal{O}(1)$ :

$K_{n}=P\left(K_{1 / 2}\right)=C^{ \pm}\left(t^{*}\right)+R\left(n\left(K_{1 / 2}\right)\right)$,

$t^{*}=t^{*}\left(K_{1 / 2}\right)$

with

$$
\begin{aligned}
C^{+}\left(t^{*}\right)= & q \int_{t_{1 / 2}}^{t^{*}} \hat{x}(t ; 0) \mathrm{d} t-I k\left(t^{*}-t_{1 / 2}\right), \\
C^{-}\left(t^{*}\right)= & q\left\{\int_{t_{1 / 2}}^{t^{*}} \hat{x}(t ; 0) \mathrm{d} t+\int_{t^{*}}^{t_{3 / 2}} \hat{a}(t ; 0) \mathrm{d} t\right\} \\
& -2 \pi I
\end{aligned}
$$

where $t^{*}=t^{*}\left(K_{1 / 2}\right)$ is given by (4.1) and (4.6) and $R\left(n\left(K_{1 / 2}\right)\right.$ ) by (3.40b). It is noted that this mapping $P(K)$ matches (3.4) for $K$ leaving the exponentially small neighborhood of $K=0$, see fig. 12.

A composite asymptotic expression for the mapping $P$ holding uniformly for $[-\pi I, \pi I]$ is constructed as follows, let $d_{3 / 2}=-A\left(t_{3 / 2}\right)$, then

$$
\begin{aligned}
& K= \pm \pi l \exp \left\{-d\left(t^{*}\right) \nu\right\}, \quad 0 \leq d \leq d_{3 / 2} \\
& P(K)=C^{ \pm}\left(t^{*}(K)\right)+R(n(K))-q K .
\end{aligned}
$$

\section{Properties of the interval mapping $P$}

\subsection{The case $\beta \in \tilde{\mathrm{A}}_{n}$}

In the discontinuous approximation we only found the stable fixed point; the unstable one is situated in the boundary layer, as is seen in fig. 13. Note that the interval has been shifted in order to

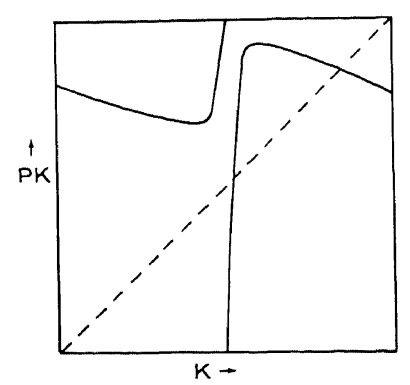

Fig. 13. The interval mapping for $\beta \in \tilde{\mathrm{A}}_{n}$. 
have $K=0$ in the interior of the interval. For any starting value different from the unstable fixed point the iterated solution will approach the stable fixed point.

\subsection{The case $\beta \in \tilde{\mathrm{B}}_{n}$}

Besides the two stable fixed points of the discontinuous approximations, there are also two unstable ones within the boundary layer. The situation is now more complicated, as it is incorrect to assume that for any starting value not coinciding with the unstable points, the iterated solution will tend to the two stable fixed points. There exists a non attracting subset of zero measure in which the iterated solution may go around in an irregular way. In order to describe this class of solutions we use symbolic dynamics, see sections 2.2 and 2.3 . As given in fig. 14 we consider subintervals $V_{i}$ ( $i=0,1,2$ and 3 ) and keep track of the mapping of points remaining in $\cup V_{i}$ in the transition matrix

$M=\left(\begin{array}{llll}0 & 1 & 1 & 1 \\ 0 & 1 & 1 & 1 \\ 1 & 0 & 0 & 0 \\ 0 & 1 & 1 & 1\end{array}\right)$

if $M_{i j}=1$ a point $\mathrm{V}_{i}$ is mapped in $\mathrm{V}_{j}$, while for $M_{i j}=0$ such a mapping is not possible. As we described in section 2.3, the topological subspace $\Sigma_{M}$ consisting of all biinfinite sequences of the symbols $0,1,2$ and 3 is introduced allowing only combinations $i j$ for which $M_{i j}=1$, i.e. forbidden combinations in the set of sequences are $00,10,21,22,23$ and 30 . It is noted that the two unstable solutions are represented as sequences of just the symbol 1 and the symbol 3, respectively. Furthermore, it is seen that the interval mapping discloses the dynamics of (1.1) to the same extent as the annulus mapping of section 2.2. The iterated solutions that correspond with an element of $\Sigma_{M}$ have zero measure. Nevertheless they give us insight in the behavior of (1.1) with starting values chosen in such a way that the solution remains in $U V_{i}$ for a large, finite number of iterations of $P$

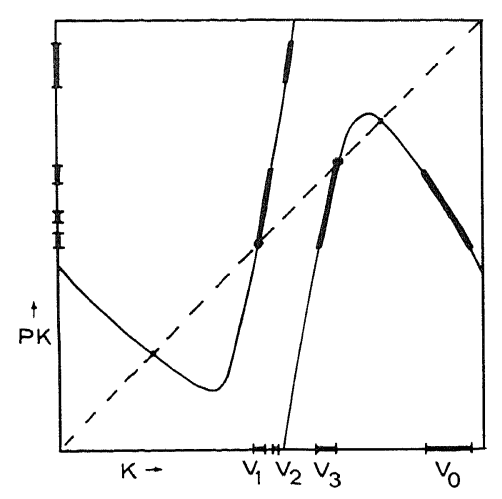

Fig. 14. The interval mapping for $\beta \in \tilde{\mathrm{B}}_{n}$.

before locking into a stable subharmonic. Initially such solutions behave in the irregular way as described here and the set of starting values has a measure different from zero.

\subsection{The transitional case $\beta \in \tilde{\mathrm{g}}_{n}$}

In the discontinuous approximation of section 4.2 a third type of structure remained out of sight. We are aiming at the case of one stable fixed point with the point $T$ below the unstable fixed point $\mathrm{S}$, see fig. 15. Then higher order stable fixed points of the iterated mapping are possible, as pointed out by Levi [11]. His statement is based on a theorem of Newhouse and Palis [20]. In fig. 15 we sketch such a solution, while in the next section we will trace one numerically in a specific example.

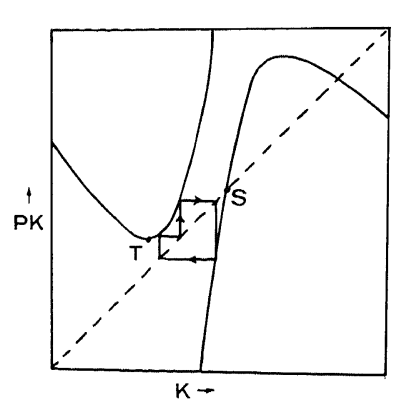

Fig. 15. The interval mapping for $\beta \in \tilde{\mathrm{g}}_{n}$. 
5.4. A numerical approximation for $\alpha=1 / 3$ and $\nu=7.5$

Using analytical and numerical methods we found for $\alpha=1 / 3$ :

$$
\begin{aligned}
I= & 3^{5 / 4}\{2 E(\arcsin \sqrt{2 / 3(1-1 / 3 \sqrt{3})}, 1 / 3 \sqrt{2-\sqrt{3}}) \\
& -F(\arcsin \sqrt{2 / 3(1-1 / 3 \sqrt{3})}, 1 / 2 \sqrt{2-\sqrt{3}}) \\
& +1 / 3(2-\sqrt{3}) \sqrt{24+14 \sqrt{3}}\} / \pi=1.47597
\end{aligned}
$$

$p=-0.061926, \quad q=0.788070, \quad \Upsilon=0.392236$,

where

$$
\begin{aligned}
& E(\phi, k)=\int_{0}^{\phi} \sqrt{1-k^{2} \sin ^{2} \theta} \mathrm{d} \theta \\
& F(\phi, k)=\int_{0}^{\phi}\left(\sqrt{1-k^{2} \sin ^{2} \theta}\right)^{-1} \mathrm{~d} \theta
\end{aligned}
$$

In order to carry out the iterations of the mapping we approximate the mapping as follows. The composite expression (4.16) is evaluated numerically in a set of points that have increasing density near $K=0$ : for $n$ points we take

$$
K(j)= \pm \pi I \exp \left\{-j \nu d_{3 / 2} / n\right\}
$$

In the computations a four point-interpolation formula is used for the points (5.4), where $P(K)$ is computed with a 6 decimal accuracy. Using this scheme we trace a stable fixed point of the second iterate by shifting the mapping in a vertical direction until in the iterated mapping two new fixed points arise, one of them being the stable fixed point we are looking for, see fig. 16. It turns out that the stable solution has a very small domain of attraction and that it is only stable over an extremely small range of $\beta$. Therefore, we only give the value of the two new fixed points, which coincide within the accuracy we are working with. They arise at

$$
K=-1.63402626
$$
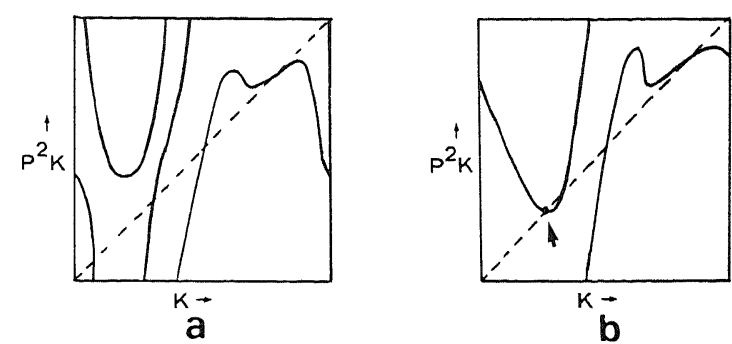

Fig. 16. The occurrence of a stable irregular subharmonic. a) A stable fixed point of period one; b) an additional stable fixed point of period two.

as $\beta$ takes one of the values

$\beta=9.3770-5.3320\left(n-\frac{1}{2}\right), \quad n=1,2, \ldots$

\section{Concluding remarks}

As a result of our asymptotic analysis, we have obtained a completely determined relation between a continuous dynamical system and a mapping on a compact interval (a one-dimensional difference equation). This result is new in the sense that up to now, there has not been yet such a description of a continuous system having chaotic type of solutions in terms of a difference equation. For the Lorenz equations [17] with its strange attractor a comparison with a system of difference equations such as the Hénon attractor is made, but the relation only refers to the occurrence of a typical set of limit points in both cases. In the study of Levi a more specific qualitative relation between a continuous system and a difference equation was established. Levi expected the interval mapping to act upon a specific domain of the state space, see section 2.2. From the present study we learn that there exists a typical difference variable which in this case turned out to be the order of a parabolic cylinder function, being part of a local asymptotic solution. It suggests that one has to look for characteristic quantities of a continuous system in order to relate it to a set of difference equations. 
From the interval mapping the bifurcation pattern is understood for the various values of $b$. For the transitional domains $\tilde{\mathrm{g}}_{k}$ the bifurcation structure turned out to be quite complex. It would be worthwhile to describe it in more detail using symbolic dynamics, as suggested in section 2 .

Constructing a numerical approximation of the interval mapping as done in section 5.4, we touched upon two points needing some consideration. Firstly, the boundary layer solution appeared to be not differentiable in the end points, which was overcome by introduction of an asymptotically small correction term. Secondly, for $\nu=7.5$ it was not possible to determine a stable irregular subharmonic for some $\beta$. From sharper calculations we concluded that even an accuracy of 14 decimals did not suffice. The reason for this is that the boundary layer in the interval mapping has a thickness of $\mathcal{O}(\exp (-\nu))$. Consequently, the graph of $P^{2}$ has an extremely sharp peak at the point where it is about to be tangent to the diagonal, see fig. 16. For stability it is necessary that $\left|\mathrm{d} P^{2} / \mathrm{d} K\right|$ $<1$ which is only the case for $\beta$ just past the point of tangency.

\section{Acknowledgement}

The authors are grateful to J. Kok of the Department of Numerical Mathematics of the CMCS for his assistance in computing numerically the functions given in figs. 7 and 8.

\section{References}

[1] J.D. Cole, Perturbation Methods in Applied Mathematics (Blaisdell, Waltham, Mass., 1968).

[2] P. Collet and J.-P. Eckmann, Iterated Maps on the Interval as Dynamical Systems (Birkhäuser, Basel, 1980).

[3] J.E. Flaherty, and F.C. Hoppensteadt, Frequency Entrainment of a Forced Van der Pol Oscillator, Stud. Appl. Math. 18 (1978) 5-15.

[4] J.P. Gollub, T.O. Brunner and B.G. Danly, Periodicity and Chaos in Coupled Nonlinear Oscillators, Science 200 (1978) 48-50.

[5] J. Grasman, Relaxation oscillations of a Van der Pol equation with large critical forcing term, Quart. Appl. Math. 38 (1980) 9-16.
[6] J. Grasman, On the Van der Pol relaxation oscillator with a sinusoidal forcing term, Mathematisch Centrum Amsterdam, Report TW 207 (1980).

[7] J. Grasman, Dips and slidings of the forced Van der Pol relaxation oscillator, Mathematisch Centrum Amsterdam, Report TW 214 (1981).

[8] J. Grasman, E.J.M. Veling and G. M. Willems, Relaxation oscillations governed by a Van der Pol equation with periodic forcing term, SIAM J. Appl. Math. 31 (1976) 667-676.

[9] J. Grasman, M.J.W. Jansen and E.J.M. Veling, Asymptotic methods for relaxation oscillations, in: Proc. Third Scheveningen Conf. on Differential Equations, W. Eckhaus and E. M. de Jager, eds., North-Holland Math. Studies, vol. 31 (North-Holland, Amsterdam, 1978), pp. 93-111.

[10] J. Guckenheimer, Symbolic dynamics and relaxation oscillations, Physica 1D (1980) 227-235.

[11] M. Levi, Qualitative analysis of the periodically forced relaxation oscillations, Mem. Amer. Math. Soc. 244 (1981).

[12] M. Levi, Periodically forced relaxation oscillations in Global Theory of Dynamical Systems, Lecture Notes in Mathematics, vol. 819, Z. Nitecki and C. Robinson, eds., (Springer, Berlin, 1980).

[13] N. Levinson, A second order differential equation with singular solutions, Ann. of Math. 50 (1949) 127-153.

[14] T.-Y. Li, and J.A. Yorke, Period three implies chaos, Amer. Math. Monthly 82 (1975) 985-992.

[15] J.E. Littlewood, On Non-Linear Differential Equations of the Second Order: III. The equation $\ddot{y}-k\left(1-y^{2}\right) \dot{y}+y$ $=b \mu k \cos (\mu t+\alpha)$ for large $k$, and its Generalisations, Acta Math. 97 (1957) 267-308.

[16] J.E. Littlewood, On Van der Pol's equation with large $k$ in Proceedings Symposium Nonlinear Problems, R.E. Langer, ed. (Univ. of Wisconsin Press, Wisconsin, 1963) pp. 161-165, 174-175.

[17] E.N. Lorenz, Deterministic nonperiodic flow, J. Atmos. Sci. 20 (1963) 130-141.

[18] R.M. May, Simple mathematical models with very complicated dynamics, Nature 261 (1976) 459-467.

[19] J. Moser, Stable and Random Motions in Dynamical Systems, Annals of Mathematics Studies, vol. 77 (Princeton Univ. Press, Princeton, N.J., 1973).

[20] S. Newhouse and J. Palis, Cycles and bifurcation theory, Astérisque 31 (1976) 43-140.

[21] D. Ruelle, Strange Attractors, Math. Intelligencer 2 (1980) 126-137.

[22] M. Shub, Stabilité globale des systèmes dynamiques, Astérisque 56 (1978) 1-211.

[23] S. Smale, Diffeomorphisms with many periodic points, in: Differential and Combinatorial Topology, Princeton Mathematical series, vol 27, S. Cairns, ed. (Princeton Univ. Press, Princeton, N.J., 1965) pp. 63-80.

[24] S. Smale, Differentiable dynamical systems, Bull. Amer. Math. Soc. 73 (1967) 747-817.

[25] P. Stefan, A. Theorem of Šarkovski on the Existence of Periodic Orbits of Continuous Endomorphisms of the Real Line, Comm. Math. Phys. 54 (1977) 237-248. 